\title{
FACTORES ASOCIADOS A LA INCIDENCIA DE TUBERCULOSIS EN UN CENTRO DE SALUD URBANO MARGINAL DE LIMA 1999-2008
}

\author{
Asociated factors to pulmonar tuberculosis incidence in a marginal urban health center of \\ Lima 1999-2008
}

Víctor Crispín ${ }^{1,2}$, Jesús Rumiche², Mirtha Roque ${ }^{2}$, Gladys Arias², José $\operatorname{Irey}^{2}$, María Salazar $^{2}$, Julio Ruiz², Andrés Herrera ${ }^{2}$, José Ortiz $^{2}$, María Carreño ${ }^{2}$, Antonio Almonacid ${ }^{2}$, Juán Pérez-León ${ }^{2}$, Patricia Crispín ${ }^{3}$, Hilda Crispín ${ }^{4}$, Felícita Abarca ${ }^{5}$.

${ }^{1}$ Centro de Salud Miguel Grau DISA IV Lima Este; ${ }^{2}$ Instituto de Investigaciones en Química Biológica, Microbiología y Biotecnología "Marco Antonio Garrido Malo" Facultad de Farmacia y Bioquímica UNMSM; ${ }^{3}$ Hospital Universitario Fundación Alcorcón, Madrid; ${ }^{4}$ City University NY; ${ }^{5}$ nstituto Nacional de Oftalmología

\section{RESUMEN}

La tuberculosis es un problema de salud pública, generado por la pobreza, con prevalencia e incidencia elevadas. El objetivo de este estudio fue determinar los factores epidemiológicos y sociales asociados a la tuberculosis en un Centro de Salud urbano marginal, desde enero 1999 hasta diciembre 2008. Es un estudio observacional, descriptivo de reporte de casos, realizado en el Centro Materno Infantil Miguel Grau, distrito de Chaclacayo, provincia de Lima. Los datos se obtuvieron del Libro de Registro de Sintomático Respiratorios y del Libro de Registro y Seguimiento de Pacientes con Tuberculosis, donde se reportaron 197 casos diagnosticados: 108 varones $(55 \%)$ y 89 mujeres $(45 \%) ; 151$ fueron nuevos $(76,6 \%)$ y 32 recaídas, abandonos recuperados y fracasos previos (16,2\%). La incidencia decreció desde 305 X 100 ooo en 1999 hasta 119 x 100 ooo habitantes el 2008. El grupo de edad más afectado fue el de $15-29$ años con 103 casos (52,3\%). Del total de casos, 174 fueron de la forma pulmonar (88\%), 176 fueron curados (89,3\%) y 14 fracasaron (7\%). En relación con los antecedentes de tuberculosis, 20 casos tenían antecedentes personales (10\%) y 106 antecedentes familiares (54\%). En 152 casos la familia estaba integrada por más de 5 miembros (77\%); 47 casos eran amas de casa (24\%), 44 estudiantes (22\%), 24 obreros (12\%) y 42 eran desocupados o sin respuesta $(21 \%)$. Se concluye que la incidencia de tuberculosis es alta, predominando la forma pulmonar con baciloscopía positiva, afectando en gran medida a la población joven; 90\% de los pacientes fueron curados. El antecedente personal o familiar de tuberculosis fue un factor frecuente; la mayoría de pacientes tenían familia mayor a 5 miembros y eran amas de casa, estudiantes, obreros o desocupados.

Palabras clave: Tuberculosis, incidencia, morbilidad, tratamiento, pobreza.

\section{SUMMARY}

Tuberculosis is a public health problem, generated by poverty, with high prevalence and incidence. The objetives of this study were to determine the associated factors to tuberculosis in a marginal urban health center of Lima. This is an observational study of case report undertaken in Chaclacayo since January 1999 to December 2008. The data collected was acquired from the routine reports of the National Control Program. A total of 197 cases were evaluated; 108 were men (55\%) and 89 were women $(45 \%)$. Within these 197 subjects, 151 were new cases $(76.6 \%)$ and 32 were individuals who had been previously diagnosed with tuberculosis (16.2\%). The incidence decreased from $305 \times 100000$ in 1999 to $119 \times 100$ ooo inhabitants in 2008. The more affected age groups were $15-29$ years with 103 cases $(52.3 \%)$. 174 patients showed a high prevalence of pulmonary tuberculosis (88\%); successful treatment were completed in 176 cases $(89,3 \%), 14$ were defaulted $(7 \%)$. 20 cases had personal antecedents (10\%) and 106 had familial antecedents (54\%). In 152 cases, the family had more than 5 members ( $77 \%)$. 47 were mistress of the house (24\%); 44 were students $(22 \%)$, 24 were laborers $(12 \%)$ and 42 were unemployed individuals or without answer (21\%). In conclusion, there were a high incidence of tuberculosis, the pulmonary form with smear positive was predominant, affecting young people, $90 \%$ of cases were cured, the personal o familial antecedent of tuberculosis was a frequent factor.

Key words: Tuberculosis, incidence, morbidity, treatment, poverty.

\section{INTRODUCCIÓN}

a Organización Mundial de la Salud (OMS) reporta que la tuberculosis (TB), enfermedad causada por Mycobacterium tuberculosis, es un problema de salud pública mun- dial, causante de 8 millones de nuevos casos y 3 millones de muertes cada año, afecta a todos los países, genera pobreza y se agrava por la aparición de cepas resistentes a fármacos de primera y segunda línea, originando la tuberculosis multidrogorresistente (TBMDR) y la tuberculosis extensivamente resistente (TB- 
XDR) ${ }^{(1,2)}$. Si no se toman medidas urgentes de control, para el período comprendido entre el año 2002 al 2020, se podrían alcanzar cifras de 1000 millones de nuevos casos de personas infectadas con $M$. tuberculosis; de los cuales 150 millones desarrollarían la enfermedad y 36 millones morirían por esta causa ${ }^{(3)}$.

En las Américas para el año 2003, de acuerdo a estimaciones de la OMS, hubieron 502605 casos prevalentes de TB, 370107 casos nuevos de todas las formas y 53803 muertes; con una tasa de incidencia estimada para de $43 \times 100$ ooo hab. para todas las formas de TB, con variaciones de 323 x 100 ooo para Haití y menos de 5 X 100.000 hab. para Estados Unidos. El Perú se ubica dentro de los países con mayor prevalencia de TB en Latinoamérica, antecedido por Haití y Bolivia ${ }^{(4,5)}$.

En el Perú, de 1994 a 2008 fueron diagnosticados entre 46 ooo y 24 ooo casos anuales de tuberculosis, situación agravada por la comorbilidad de infección por el virus de la inmunodeficiencia humana (TB-VIH SIDA), la TB-MDR y la TB-XDR. Tres a cuatro personas $\mathrm{x}$ hora enfermaron de TB, 81 casos $\mathrm{x}$ día, más de 3 muertes $\mathrm{x}$ día, 1,053 muertes $\mathrm{x}$ año, en promedio. El $58 \%$ de casos de TB, $82 \%$ de casos de TB MDR y $93 \%$ de casos de TB XDR son notificados en Lima y Callao; en Lima Este las zonas de mayor riesgo se encuentran en los distritos de San Juan de Lurigancho, Ate, El Agustino y Santa Anita ${ }^{(6,7)}$.

Esta situación configura un futuro poco alentador y obliga en todos los niveles a replantear las estrategias epidemiológicas para su control basado en el conocimiento de la realidad.

El propósito del presente estudio fue analizar los factores asociados a la incidencia de la tuberculosis y su evolución en los últimos 10 años en una zona urbano marginal de Lima.

\section{MATERIALES Y MÉTODOS}

El presente es un estudio observacional, descriptivo, retrospectivo, realizado en el Centro Materno Infantil (CMI) Miguel Grau, centro base de la microrred Chaclacayo, de la región de salud IV Lima Este. A dicho centro acuden los pobladores de la comunidad de Miguel Grau, la cual se ubica en la ribera izquierda del río Rímac, en el distrito de Chaclacayo, provincia de Lima, con altitud de $647 \mathrm{msnm}$, de clima seco y templado, con temperatura variable según la estación, con importantes vías de comunicación como el ferrocarril y la carretera central. El área corresponde a una zona urbano marginal, con una población de 11,978 habitantes, con viviendas de ladrillo y cemento, con servicios básicos, con población adulta mayor en su mayoría migrante del interior del país, de ocupación obreros, artesanos y choferes ${ }^{(8)}$.

La población estudiada la constituyeron todos los pacientes que fueron diagnosticados de tuberculosis en el CMI Miguel Grau, desde enero 1999 hasta diciembre 2008 y que recibieron tratamiento antituberculoso con medicamentos de los esquemas establecidos por el Programa de Control de Tuberculosis del Ministerio de Salud (actualmente Estrategia Sanitaria Nacional de Prevención y Control de Tuberculosis) ${ }^{(9)}$.

Los datos se obtuvieron de los libros que el programa de control utiliza para el diagnóstico (Libro de Sintomático Respiratorios) y seguimiento (Libro de Registro y Seguimiento de pacientes con tuberculosis), y fueron corroborados a través de la Tarjeta de control de medicamentos de los pacientes de los años respectivos.

Un caso de tuberculosis fue definido como "todo paciente a quién se diagnostica tuberculosis, con o sin confirmación bacteriológica, y a quien se decide indicar y administrar un tratamiento antituberculoso". Según se trate de ${ }^{\left({ }^{9}\right.}$ :

- Caso nuevo, aquel paciente diagnosticado por primera vez y que por tanto nunca ha recibido tratamiento específico.

- Recaída, aquel paciente que presenta un nuevo episodio de tuberculosis después de haber completado un tratamiento exitoso (haber sido curado) y permanece sano por un tiempo mayor a seis meses.

- Abandono recuperado, aquel paciente que luego de iniciar tratamiento, no concurrió a recibirlo por más de treinta días consecutivos y luego de este tiempo reingresa al programa.

- Fracaso, aquel paciente que mantiene baciloscopías positivas hasta el cuarto mes de tratamiento supervisado o aquellos que presentan baciloscopías positivas en dos controles sucesivos después de un período de negativización de dos meses en un esquema supervisado.

Para el análisis de los datos se realizó la digitación en hojas de cálculo Excel versión 3.o. Los resultados y gráficos que se muestran se obtuvieron del mismo programa. Se definió la incidencia como el número de casos nuevos de tuberculosis sobre la población total para cada año de estudio ajustada por 100 ooo habitantes.

\section{RESULTADOS}

Durante el período de estudio y mediante criterios epidemiológicos, clínicos, radiológicos y microbiológi- 
cos (baciloscopía y cultivo) fueron diagnosticados 197 casos de tuberculosis, 108 varones $(55 \%)$ y 89 mujeres ( $45 \%)$, de los cuales 151 casos eran nuevos $(77 \%), 29$ fueron por recaídas y abandonos recuperados (15\%), 3 fueron fracasos a esquemas previos (1\%) y los restantes 14 fueron transferencias recibidas (7\%) (Tablas 1 y 2 ).

La incidencia de tuberculosis (TB) disminuyó paulatinamente desde $305 \mathrm{x} 100$ ooo en 1999 hasta 119 x 100 ooo habitantes el 2008. Igualmente, la incidencia de la tuberculosis pulmonar esputo positivo (TBP $\mathrm{BK}(+))$ disminuyó desde $229 \times 100$ ooo en 1999 hasta 76 x 100 ooo habitantes el 2008 (Figura 1).

Los grupos etarios más afectados fueron de 15 -19 años con 35 casos (17,8\%), 20-24 años con 39 casos $(19,8 \%)$ y $25-29$ años con 29 casos (14,7\%), sumando $52.3 \%$ de los casos. El grupo de 3044 años comprendió 53 casos $(27 \%), 15$ casos $7,6 \%$ eran mayores de 60 años (Tabla 3 ).

La forma pulmonar de tuberculosis fue la más frecuente con 174 casos (88\%), frente a 23 pacientes de la forma extra-pulmonar (12\%). De los casos con tuberculosis pulmonar, 147 fueron baciloscopía positiva (74\%) de los cuales 31 casos fueron BK (+++) (16\%), 7 tuvieron cultivo positivo (4\%) y, 20 tenían baciloscopía negativa y radiografía de tórax compatible con tuberculosis pulmonar (10\%) (Tabla 4).

La condición general de egreso de los pacientes fue de 176 curados $(89,3 \%), 14$ fracasos $(7 \%)$, 2 abandonos $(1 \%), 2$ fallecidos durante el tratamiento $(1 \%)$ y en 3 casos $(1,5 \%)$ se presentaron reacciones adversas a fármaco antituberculoso (RAFA). De 151 pacientes trata-

dos con el esquema uno, fueron curados 137 (92\%) y 7 fracasaron (4.7\%); de 6 casos tratados con el esquema dos reforzado, fueron curados $4(67 \%)$ y 2 fracasaron (33\%); de 29 casos tratados con el esquema dos, fueron curados 24 ( $83 \%$ ) y 5 fracasaron (17\%) y, de los 11 casos tratados con el esquema tres, fueron curados el $100 \%$ (Tabla 5).

Del total de casos, 126 tenían antecedentes de tuberculosis $(64 \%), 20$ con antecedentes personales (10\%) y 106 con antecedentes familiares (54\%) (Tabla 6). En 45 casos la familia estaba integrada por 1 a 4 miembros (23\%) y en 152 , de 5 a más miembros ( $77 \%)$ (Tabla 7 ).

De los pacientes con tuberculosis pulmonar 47 eran amas de casa (24\%), 44 eran estudiantes (22\%), 24 eran obreros $(12,5 \%)$ y 42 del grupo desocupados o sin respuesta (21\%) (Figura 2).

Tabla 1. Casos de tuberculosis según género durante el período 1999 - 2008

\begin{tabular}{|c|c|c|c|c|c|c|c|c|c|c|c|c|}
\hline \multirow{2}{*}{ Género } & \multicolumn{10}{|c|}{ Años de estudio } & \multicolumn{2}{|c|}{ Total } \\
\hline & 1999 & 2000 & 2001 & 2002 & 2003 & 2004 & 2005 & 2006 & 2007 & 2008 & № & $\%$ \\
\hline Varones & 12 & 17 & 13 & 14 & 12 & 13 & 7 & 9 & 7 & 4 & 108 & 55 \\
\hline Mujeres & 16 & 8 & 9 & 9 & 8 & 8 & 7 & 9 & 5 & 10 & 89 & 45 \\
\hline Total & 28 & 25 & 22 & 23 & 20 & 21 & 14 & 18 & 12 & 14 & 197 & 100 \\
\hline
\end{tabular}

Tabla 2. Condición de ingreso de los pacientes 1999 - 2008

\begin{tabular}{lcccccccccccccc}
\hline \multicolumn{1}{c}{$\begin{array}{c}\text { Condición de } \\
\text { ingreso }\end{array}$} & \multicolumn{1}{c}{} & $\mathbf{1 9 9 9}$ & $\mathbf{2 0 0 0}$ & $\mathbf{2 0 0 1}$ & $\mathbf{2 0 0 2}$ & $\mathbf{2 0 0 3}$ & $\mathbf{2 0 0 4}$ & $\mathbf{2 0 0 5}$ & $\mathbf{2 0 0 6}$ & $\mathbf{2 0 0 7}$ & $\mathbf{2 0 0 8}$ & Total & $\mathbf{0}$ \\
\hline Nuevos & 21 & 18 & 15 & 20 & 15 & 14 & 14 & 15 & 5 & 14 & 151 & 77 \\
Recaíd-Aband-Recup & 3 & 4 & 5 & 1 & 4 & 4 & 0 & 3 & 5 & 0 & 29 & 15 \\
Fracasos & 1 & 1 & 1 & 0 & 0 & 0 & 0 & 0 & 0 & 0 & 3 \\
Transfer Recibidas & 3 & 2 & 1 & 2 & 1 & 3 & 0 & 0 & 2 & 0 & 14 & 7 \\
Total & 28 & 25 & 22 & 23 & 20 & 21 & 14 & 18 & 12 & 14 & 197 & 100 \\
\hline
\end{tabular}


Tabla 3. Casos de tuberculosis por grupo etario y sexo 1999-2008

\begin{tabular}{|c|c|c|c|c|c|c|c|c|c|c|c|c|c|}
\hline \multirow{2}{*}{ Sexo } & \multicolumn{12}{|c|}{ Grupo etario } & \multirow{2}{*}{ Total } \\
\hline & $0-9$ & $10-14$ & 15-19 & $20-24$ & $25-29$ & $30-34$ & $35-39$ & $40-44$ & 45-49 & $50-54$ & 55-59 & $>60$ & \\
\hline Varones & 0 & 6 & 16 & 18 & 19 & 14 & 12 & 7 & 2 & 5 & 2 & 7 & 108 \\
\hline Mujeres & 1 & 2 & 19 & 21 & 10 & 7 & 6 & 7 & 3 & 4 & 1 & 8 & 89 \\
\hline Total & 1 & 8 & 35 & 39 & 29 & 21 & 18 & 14 & 5 & 9 & 3 & 15 & 197 \\
\hline$\%$ & 0,5 & 4,1 & 17,8 & 19,8 & 14,7 & 10,7 & 9,1 & 7 & 2,5 & 4,6 & 1,5 & 7,6 & 100 \\
\hline
\end{tabular}

Tabla 4. Formas de Tuberculosis y Resultado de las Pruebas de Laboratorio

\begin{tabular}{|c|c|c|c|c|c|c|c|c|c|c|c|c|}
\hline \multirow{2}{*}{ Forma de tuberculosis } & \multicolumn{10}{|c|}{ Años de estudio } & \multicolumn{2}{|c|}{ Total } \\
\hline & 1999 & 2000 & 2001 & 2002 & 2003 & 2004 & 2005 & 2006 & 2007 & 2008 & № & $\%$ \\
\hline Pulmonar BK (-) & 5 & 0 & 0 & 2 & 2 & 5 & 0 & 1 & 0 & 5 & 20 & 10 \\
\hline Pulmonar BK (+) & 7 & 14 & 11 & 7 & 7 & 8 & 5 & 7 & 0 & 4 & 70 & 35 \\
\hline Pulmonar BK (++) & 9 & 5 & 4 & 3 & 6 & 2 & 6 & 3 & 5 & 3 & 46 & 23 \\
\hline Pulmonar BK $(+++)$ & 4 & 3 & 2 & 5 & 2 & 2 & 2 & 3 & 6 & 2 & 31 & 16 \\
\hline Cultivo BK (+) & 1 & 1 & 1 & 2 & 0 & 1 & 0 & 1 & 0 & 0 & 7 & 4 \\
\hline Extrapulmonar & 2 & 2 & 4 & 4 & 3 & 3 & 1 & 3 & 1 & 0 & 23 & 12 \\
\hline Total & 28 & 25 & 22 & 23 & 20 & 21 & 14 & 18 & 12 & 14 & 197 & 100 \\
\hline
\end{tabular}

\section{DISCUSIÓN}

La Tuberculosis (TB) es una de las enfermedades infecciosas transmisibles de alta incidencia en el mundo, no obstante ser potencialmente prevenible y curable, el 95\% de los enfermos y el 98\% de las defunciones se ubican en los países en desarrollo, y continúa siendo un gran problema de salud pública, sobre todo en las zonas de mayor pobreza y de hacinamiento ${ }^{(6,10)}$.

El control de la TB requiere el diagnóstico precoz y el tratamiento adecuado de los pacientes, para disminuir la transmisión de la enfermedad, lo que implica altos costos económicos para su control y costos indirectos en los que incurre el individuo y la sociedad.

Durante el período de estudio, desde 1999 hasta 2008, mediante los antecedentes epidemiológicos, el examen clínico, las imágenes radiológicas, los resultados de la baciloscopía y el cultivo del Mycobacterium tuberculosis, fueron diagnosticados 197 casos de tuberculosis, afectando más a los varones $(55 \%)$ que a las mujeres (45\%) (Tabla 1), diferencia que puede explicarse por el mayor grado de interacciones sociales de los varones en las actividades públicas ${ }^{(11,12)}$.

El número de casos tuvo tendencia a disminuir paulatinamente, lo cual se refleja en la incidencia de la tuberculosis (TB) que disminuyó de 305 x 100 ooo en 1999 hasta 119 x 100 ooo habitantes el 2008, y en la incidencia de la tuberculosis pulmonar esputo positivo (TBP BK(+)), que disminuyó desde $229 \times 100$ ooo en 1999 hasta 76 x 100 ooo habitantes el 2008 (Figura 1). Estos datos evidencian una disminución del $39 \%$ y $33 \%$, de la TB y TBP BK(+), respectivamente. En general la incidencia tiene una tendencia a disminuir en el centro de estudio, pero siempre fue mayor a las incidencias de la región de salud Lima Este y del Perú, tanto para TB como de TBP BK (+); y los porcentajes de disminución también son menores con relación a los datos nacionales. De tal manera, que la tuberculosis continúa siendo un problema de salud pública ${ }^{(6,9)}$.

En relación con la condición de ingreso al tratamiento, la mayoría de los pacientes eran casos nuevos nunca antes tratados $77 \%$, seguido por las recaídas y abandonos recuperados $15 \%$, las transferencias recibidas $7 \%$ y los fracasos a tratamientos previos $1,5 \%$ (Tabla 2).

Se sabe que la tuberculosis afecta a personas de todas las edades, sobre todo a jóvenes y adultos como se observó en nuestro estudio, donde $52.3 \%$ de los casos tenían de 15 a 29 años, $26,8 \%$ tenían de 30 a 44 años y 7,6\% eran mayores de 60 años (Tabla 3), es decir, en el CMI Miguel Grau al igual que en otras regiones del Perú, esta enfermedad afecta a la población económicamente activa siendo una enfermedad generadora de pobreza ${ }^{(6,11,13)}$.

La forma pulmonar de TB, crónica, broncogénica o tisis, es la forma más prevalente de la enfermedad, la primera causa de muerte de los enfermos con TB, y es responsable de la diseminación de la enfermedad ${ }^{(11,13)}$; esta forma incluye a los casos con baciloscopía positiva y negativa y los que tuvieron cultivo positivo. En nuestro estudio se presentó en $88 \%$ de los pacientes.

La baciloscopía es la prueba de laboratorio más económica y rápida para el diagnóstico de la TBP, consiste en evidenciar la presencia de los bacilos alcohol ácido-resistentes (BAAR) en el esputo ${ }^{(9)}$, como sucedió en $75 \%$ de los pacientes. Al mismo tiempo los 
Tabla 5. Resultado del tratamiento de los pacientes

\begin{tabular}{|c|c|c|c|c|c|c|c|c|c|c|}
\hline \multirow{3}{*}{ Resultado } & \multicolumn{10}{|c|}{ № de Pacientes según Esquema de Tratamiento* } \\
\hline & \multicolumn{2}{|c|}{ Uno } & \multicolumn{2}{|c|}{ Dos-R } & \multicolumn{2}{|c|}{ Dos } & \multicolumn{2}{|c|}{ Tres } & \multicolumn{2}{|c|}{ Total } \\
\hline & № & $\%$ & № & $\%$ & № & $\%$ & № & $\%$ & № & $\%$ \\
\hline Curados & 137 & 91 & 4 & 67 & 24 & 83 & 11 & 100 & 176 & 89,3 \\
\hline Fracasos & 7 & 5 & 2 & 33 & 5 & 17 & 0 & 0 & 14 & 7,2 \\
\hline Abandonos & 2 & 1 & 0 & 0 & 0 & 0 & 0 & 0 & 2 & 1 \\
\hline Fallecidos & 2 & 1 & 0 & 0 & 0 & 0 & 0 & 0 & 2 & 1 \\
\hline RAFA & 3 & 2 & 0 & 0 & 0 & 0 & 0 & 0 & 3 & 1,5 \\
\hline Total & 151 & 100 & 6 & 100 & 29 & 100 & 11 & 100 & 197 & 100 \\
\hline
\end{tabular}

* Esquemas de tratamiento: Uno: $2 \mathrm{HRZE} / 4 \mathrm{H}_{2} \mathrm{R}_{2}$ Dos: $2 \mathrm{HRZES} / 1 \mathrm{HRZE} / 5 \mathrm{H}_{2} \mathrm{R}_{2} \mathrm{E}_{2}$ Dos-R: $3 \mathrm{HRZES} / 5 \mathrm{H}_{2} \mathrm{R}_{2} \mathrm{E}_{2} \mathrm{~S}_{2}$ (años 1996-1998) Tres: $2 \mathrm{HRZ} / 3 \mathrm{H}_{2} \mathrm{R}_{2}(\mathrm{Hasta} 2001)$

Tabla 6. Antecedentes de tuberculosis de los pacientes

\begin{tabular}{lcc}
\hline \multicolumn{1}{c}{ Antecedente } & No & \% \\
\hline Personal & 20 & 10 \\
Familiar: & & \\
Esposo(a) & 8 & 4 \\
Padres & 26 & 13 \\
Hermano(a) & 31 & 16 \\
Hijo(a) & 10 & 5 \\
Otro & 31 & 16 \\
Ninguno & 71 & 36 \\
Total & 197 & 100 \\
\hline
\end{tabular}

Tabla 7. Número de miembros de familia

\begin{tabular}{lcc}
\hline \multirow{2}{*}{$\begin{array}{c}\text { Número de } \\
\text { miembros }\end{array}$} & \multicolumn{2}{c}{ Pacientes } \\
\cline { 2 - 3 } $1-4$ & No & \% \\
\hline $1-4$ & 45 & 23 \\
$5-9$ & 132 & 67 \\
$>9$ & 20 & 10 \\
Total & 197 & 100 \\
\hline
\end{tabular}

de $85 \%$ de los pacientes) para dicho esquema ${ }^{(14)}$, con cifras mínimas de fracasos $5 \%$, abandonos $1 \%$ y fallecidos $1 \%$. Tres pacientes $(2 \%)$ presentaron reacción adversa (RAFA) y fue necesario el cambio a un esquema individualizado. Cuando los medicamentos del esquema Uno son administrados siguiendo estrictamente la estrategia del tratamiento acortado directamente observado (DOTS), los resultados son buenos para el paciente, su familia y la comunidad ${ }^{(15)}$. De los 29 pacientes tratados con el esquema Dos fueron curados $83 \%$ y fracasaron $17 \%$, en donde se observa que los pacientes con recaídas tienen menor probabilidad de curación. De los 11 pacientes tratados con el esquema Tres fueron curados el $100 \%$. Los esquemas de tratamiento son objeto de evaluación permanente según eficacia y eficiencia, así, fue retirado el esquema Dos-Reforzado que se aplicó para los fracasos al esquema Uno, por tener bajos niveles de curación, que en nuestro caso, no obstante el reducido número de pacientes - seis - sólo fueron curados $4(67 \%)$ y fracasaron 2 (33\%). Estas cifras aplicadas al nivel regional o nacional, pueden considerarse como una de las causas del elevado número de casos de TB-MDR ${ }^{(15)}$. Al evaluar la eficacia del programa de control de tuberculosis en el CMI M. Grau, para el período de estudio, las cifras de diagnóstico de TBP BK(+) (74\%) y de curación de la enfermedad $(89,3 \%)$ superan las metas de la OMS $(70 \%$ de detección y $85 \%$ de curación) ${ }^{(14)}$ (tablas 4 y 5 ).

$\mathrm{Al}$ investigar los antecedentes epidemiológicos se encontró que $64 \%$ de los pacientes tenían antecedentes de tuberculosis, $15 \%$ ingresaron por recaída, abandono o recuperación y $54 \%$ tenían antecedentes familiares, por haber sido contactos (Tabla 6). Así, el antecedente epidemiológico de la enfermedad es un factor determinante. En la zona de estudio, la forma predominante de tuberculosis fue la pulmonar, como ocurre en las 


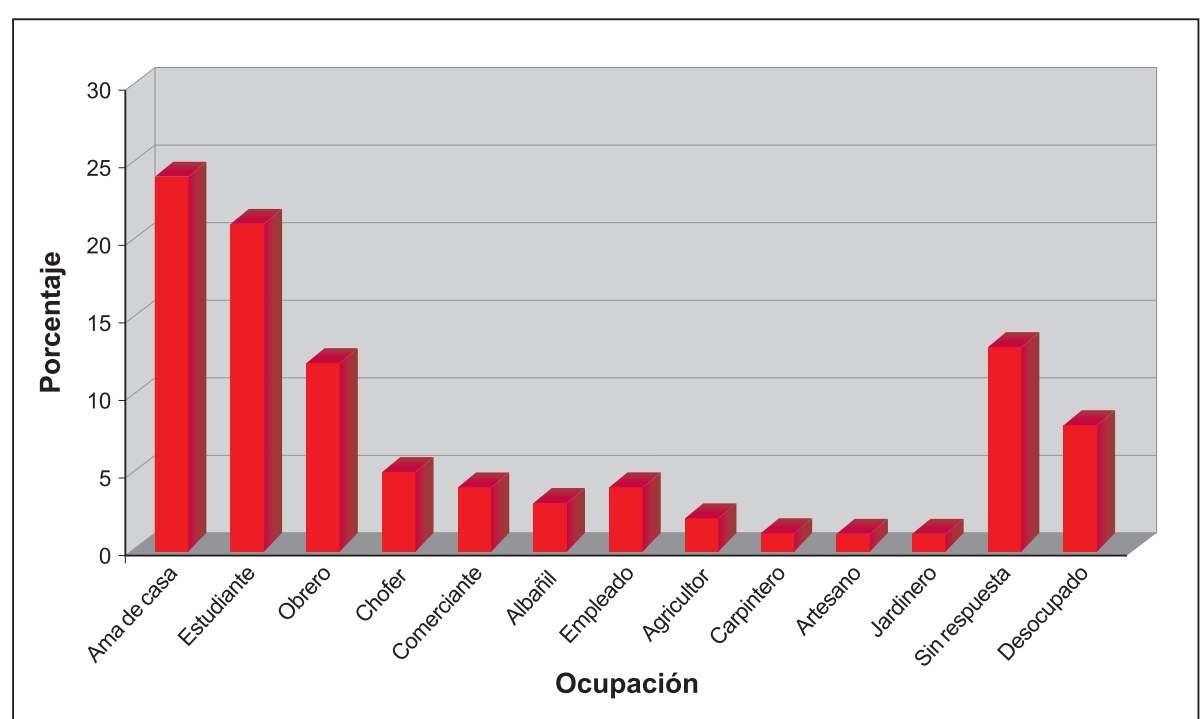

Figura 2. Ocupación de los pacientes con tuberculosis en el CMI Miguel Grau DISA IV LE 1999 - 2008 ingreso, el trabajo, la vivienda y el nivel de educación (determinantes sociales de la salud). La medicina y los servicios de salud constituyen sólo uno de los factores que tiene influencia sobre la salud de la población. En realidad, los factores principales se hallan en el amplio espectro de condiciones sociales y económicas en que vive la gente: la pobreza en sus diversas manifestaciones, las injusticias, el déficit de educación, deficiencias en la nutrición, la marginación social, la protección insuficiente de la infancia temprana, la discriminación contra la mujer, la vivienda insalubre, el deterioro urba- diferentes localidades y regiones del Perú, esta es la forma contagiosa de la enfermedad, la misma que se puede transmitir al conversar, estornudar, bostezar, es decir, entre las personas cercanas al enfermo (contactos), por lo cual en mayor riesgo se encuentran en primer lugar los familiares y los compañeros de estudio o trabajo ${ }^{(12)}$.

Los factores socioeconómicos desempeñan una función importante para definir el nivel de vida y determinar el comportamiento de los índices epidemiológicos en distintos países. La tuberculosis aparece en donde existe pobreza, desnutrición y carencia de atención médica adecuada ${ }^{(16)}$. La pobreza condiciona la falta de vivienda adecuada con situación de hacinamiento como es el caso del CMI Miguel Grau donde $77 \%$ de casos tenían familia con más de 5 miembros (Tabla 7).

Otros factores determinantes para la incidencia de la tuberculosis, además de la pobreza, son la migración, la preponderancia de estilos de vida no saludables, los factores demográficos, las restricciones en la accesibilidad a los servicios de salud, así como la condición de los ambientes laborales (17).

Se ha sostenido que las desigualdades en la distribución de la tuberculosis están relacionadas con distintos indicadores sociales como el desempleo y la conflictividad social. En nuestro estudio $24 \%$ de los pacientes con tuberculosis eran amas de casa, $22 \%$ estudiantes, $\mathbf{1 2 . 5} \%$ obreros, $21 \%$ eran del grupo desocupados o sin respuesta (Figura 2).

La desigualdad en salud está en relación con el no, la falta de agua potable, la violencia generalizada, las brechas y disparidad en los sistemas de seguro social. Se ha descrito una estrecha asociación entre la pobreza y enfermedad, las causas de las enfermedades son tanto sociales y económicas, como físicas, y se ha recomendado, como remedio: prosperidad, educación y libertad, en un marco de democracia libre e ilimitada ${ }^{(17,18,19)}$.

\section{CONCLUSIONES}

Durante el período de estudio la incidencia de la TB disminuyó desde 305 x 100 ooo hasta 119 x 100 ooo habitantes, la forma pulmonar fue la más frecuente (88\%), la baciloscopía fue positiva en $74 \%$ de los casos, aún cuando el diagnóstico fue tardío en 39\% de los pacientes; enfermaron principalmente población económicamente activa (15 a 49 años). La tasa de curación para el esquema Uno fue 91\%, pero al considerar los otros esquemas la tasa disminuyó a 89,3\%; 64\% de los pacientes tenían antecedente de tuberculosis. Otros factores asociados fueron el hacinamiento y ser ama de casa o estudiante.

\section{REFERENCIAS BIBLIOGRÁFICAS}

1. World Health Organization. Global tuberculosis control: surveillance, planning, financing. WHO report 2007. Geneva, World Health Organization, 2007 (WHO/HTM/TB/2007.376). Disponible en: www.who. int/tb/publications/global_report/2007/pdf/full.pdf Consultado el 02-10-2010 (21:43).

2. Aguilar M, Cortés C, Zenteno R. Conocimiento y actitudes sobre tuberculosis en personal médico de Veracruz, México. Med UNAB 2008; 11(3): 213-7. 
3. World Health Organization. Global tuberculosis control: surveillance, planning, financing. WHO report 2004. Geneva, World Health Organization, 2004 (WHO/HTM/TB/2004.331). Disponible en: www.who. int/tb/publications/global_report/2004/contents.pdf. Consultado el 02-10-2010 (21:49).

4. World Health Organization. Global tuberculosis control: surveillance, planning, financing. WHO report 2005. Geneva, World Health Organization (WHO/HTM/ TB/2005.349). Disponible en: www.who.int/tb/publications/global_report/2005/pdf/Full.pdf. Consultado el 01-10-2010 (22:10).

5. Organización Panamericana de la Salud La Salud en las Américas: 2007 Washington, DC: OPS, 2007-2v. (OPS, Publicación Científica y Técnica No. 622) ISBN 9789275 31626 o (Volumen I- Regional).

6. Bonilla AC. Situación de la tuberculosis en el Perú. Acta Med Per 2008; 25(3): 163-70.

7. Ministerio de Salud. Situación de Salud 2009 Dirección de Salud IV Lima Este. Lima 2009.

8. Dirección de Salud IV Lima Este. Análisis de la Situación de Salud 2008 CMI Miguel Grau DISA IV LE. Lima, 2008.

9. Ministerio de Salud. Norma técnica de salud para el control de la tuberculosis / Ministerio de Salud. Dirección General de Salud de las Personas. Estrategia Sanitaria Nacional de Prevención y Control de la Tuberculosis Lima: Ministerio de Salud; 2006.

10. Said-Fernández S, Becerril-Montes P, Molina-Salinas G, Barrios-García H, Vargas-Villarreal J. Tuberculosis causada por cepas de Mycobacterium tuberculosis drogorresistentes. Enf Emerg 2005; 7(1): 13-9.

11. Farga V. Tuberculosis 2a. ed. Publ. Téc. Mediterráneo Ltda. Santiago de Chile, 1992.

12. Beggs C, Noakes C, Sleigh P, Fletcher L, Siddiqi K. The transmission of tuberculosis in confined spaces: an analytical review of alternative epidemiological models. Int J Tuberc Lung Dis 2003; 7(11): 1015-26.

13. Rieder H. Bases epidemiológicas del control de la tuberculosis ira. ed UITER París 1999.

14. World Health Organization. Compendium of Indicators for Monitoring and Evaluating National
Tuberculosis Programs WHO/HTM/TB/2004.344 Stop TB Partnerships Geneve, 2004. Disponible en: whqlibdoc.who.int/hq/2004/WHO_HTM_TB_2004.344.pdf.

Consultado el o2-10-2010 (21:15).

15. Jave O. Investigando en tuberculosis. ¿Dónde estamos, quiénes somos, hacia dónde nos dirigimos? Rev Peru Med Exp Salud Publica. 2009; 26(3): 276-77.

16. Gutiérrez DAG, Gutiérrez PKJ, Toledo CFJ. Factores asociados a la incidencia de tuberculosis en personas de 15 a 49 años residentes en los distritos V y VI Municipio de Tipitapa del 1 de enero del 2003 al 30 de junio del 2004. Tesis Maestría en Salud Pública. Univ. Nac. Auton. de Nicaragua Managua, 2005.

17. Díaz de Quijano E, Brugal M, Pasarín M, Galdós-Tangüís $\mathrm{H}$, Cayla J, Borrel C. Influencia de las desigualdades sociales, la conflictividad social y la pobreza extrema sobre la morbilidad por tuberculosis en la ciudad de Barcelona. Rev Esp Salud Publica. 2001; 75(6): 517-28.

18. Rojas F. El componente social de la salud pública en el siglo XXI. Rev Cub Salud Publica [online] 2004; 30(3) [citado 2010-09-27], pp. o-o . Disponible en: <http:// scielo.sld.cu/scielo.php?script=sci_arttext\&pid=So864$34662004000300008 \& \operatorname{lng}=\mathrm{es} \& n \mathrm{~mm}=\mathrm{iso}>$. ISSN o8643466.

19. Berlinguer G. Determinantes sociales de las enfermedades. Rev Cub Salud Publica [online] 2007; 33(1) [citado 2010o9-27], pp. o-o. Disponibleen:<http://scielo.sld.cu/scielo. php?script=sci_arttext\&pid=So864-34662007000100003\&lng=es\&nrm=iso $>$. ISSN o864-3466

Manuscrito recibido el: 26/o1/2010

Aceptado para su publicación el: 02/07/2010

\section{Correspondencia:}

Nombre: Víctor Crispin Pérez

Dirección: Ca. Santa Verónica № 233 - Lima 31 / Jr. Puno 1002 - Lima o1 - Perú

e-mail:vcrispinp@hotmail.com 